\title{
Balanceamento de Carga Inspirado no Comportamento das Abelhas Aplicado a uma Rede Perinatal
}

\author{
Hudson H. S. Lopes, Ricardo B. O. de Oliveira, Flávio G. C. Rocha \\ Escola de Engenharia Elétrica, Mecânica e de Computação \\ Universidade Federal de Goiás (UFG) \\ Caixa Postal 74605-010 - Goiânia - GO - Brasil \\ hudson.hsl@gmail.com, ricfire.ricardo@gmail.com, flaviogcr@ufg.br
}

\begin{abstract}
This paper considers the problem of efficient allocation of resources in the admission and scheduling process of pregnant women to a perinatal network composed of two maternities. For this, a load balancing algorithm based on the behavior of bees is used. It is shown, through the results obtained in the simulations, that the algorithm reduces the waiting time for pregnant women who enter the perinatal system, in comparison with other load-balancing algorithms found in the literature.
\end{abstract}

Resumo. Neste artigo considera-se o problema de alocação eficiente de recursos no processo de admissão e escalonamento de gestantes para uma rede perinatal composta por duas maternidades. Para tanto, utiliza-se um algoritmo de balanceamento de carga baseado no comportamento das abelhas. Apresenta-se, por meio dos resultados obtidos nas simulações, que o algoritmo reduz o tempo de espera das gestantes que entram no sistema perinatal, em comparação a outros algoritmos de balanceamento de carga presentes na literatura.

\section{Introdução}

Por perinatal entende-se tudo o que acontece entre a $22^{\mathrm{a}}$ semana de gestação e a primeira semana de vida do recém-nascido. As unidades de saúde perinatal no Brasil são responsáveis por esses períodos e enfrentam múltiplos desafios atualmente [do Carmo Leal et al. 2015]. Diante desses desafios encontrados pelo sistema de saúde em geral, muitos projetos de pesquisa aplicados à saúde têm sido desenvolvidos em vários campos da ciência. Green [Green 2005] e Pehlivan [Pehlivan 2014] descreveram os antecedentes gerais e as questões envolvidas no planejamento da capacidade hospitalar e mostraram exemplos de como as metodologias de Pesquisa Operacional podem ser usadas para fornecer informações importantes sobre estratégias e práticas operacionais.

Este artigo propõe a aplicação de um algoritmo de balanceamento de carga inspirado no comportamento das abelhas para um sistema perinatal composto por duas unidades de atendimento com capacidades heterogêneas. O Algoritmo tem por objetivo diminuir o tempo médio de trabalho e consequentemente o tempo de espera das gestantes no cenário proposto.

\section{O Sistema Maternidade}

O funcionamento do sistema da maternidade é detalhado na Figura 1, quando uma gestante chega à maternidade ela passa por um processo onde são verificadas as condições 
relacionadas ao parto (triagem). Na sala de pré-parto a equipe médica determina com base na avaliação do quadro clínico da gestante se o parto será cirúrgico ou não cirúrgico. Os partos cirúrgicos do tipo cesariana são realizados no Centro Cirúrgico (CC), os partos não cirúrgicos do tipo normal são realizados no Centro Obstétrico (CO). Após o parto, processa-se a saída da gestante do CC ou CO e seu encaminhamento para o leito pósparto, em casos com risco de vida para a gestante e/ou ao recém-nascido eles podem ser deslocados para a UTI, onde permanecem até que se recuperem totalmente.

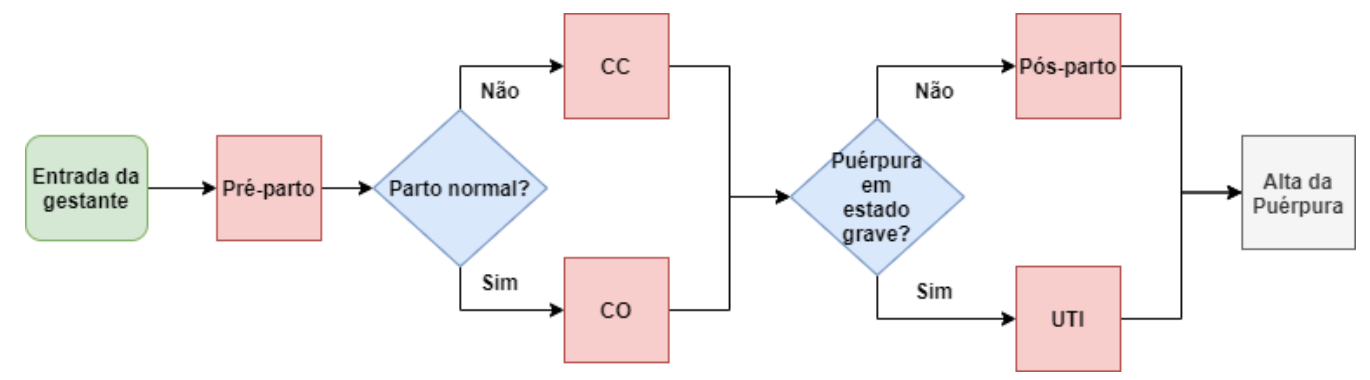

Figura 1. Sistema de uma Maternidade

\section{Balanceamento de Carga Inspirado no Comportamento das Abelhas}

Inspirado pelo comportamento inteligente das colônias de abelhas definido pelo forrageamento ou busca por alimento dos enxames de abelhas produtoras de mel, Karaboga propôs em [Karaboga 2005] o algoritmo Artificial Bee Colony Algorithm (ABC), uma meta-heurística baseada em população. Babu e Krishna em [Babu and Krishna 2013] desenvolveram um algoritmo para balanceamento de carga de tarefas em ambiente de computação em nuvem (HBB-LB) (do inglês, Honey bee behavior inspired load balancing).

A admissão de uma gestante em uma maternidade pode ser comparada a uma abelha que procura uma fonte de alimento. Quando uma maternidade está sobrecarregada, ou seja, o serviço de atendimento sendo utilizado é semelhante ao alimento sendo esgotado em uma fonte, neste caso, a gestante será transferida para uma maternidade com maior capacidade de atendimento, semelhante a uma abelha operária que encontra uma nova fonte de alimento. A informação da transferência dessa gestante é uma etapa análoga à dança executada pelas abelhas operárias ao informarem as abelhas forrageiras que encontraram alimento. Esta atualização dará subsídios para tomada de decisão de qual maternidade a gestante deve ser admitida com base na sua capacidade.

\subsection{Modelo da Meta-Heurística Aplicada ao Sistema Perinatal}

O modelo matemático é apresentado da seguinte forma: seja $\mathrm{M}=\left\{M_{1}, M_{2}, \ldots, M_{m}\right\} \mathrm{o}$ conjunto de $m$ maternidades e $\mu=\left\{\mu_{1}, \mu_{2}, \ldots, \mu_{m}\right\}$, o conjunto com as suas $m$ respectivas taxas de atendimento, que deverão atender $n$ gestantes representado pelo conjunto $\mathrm{G}=$ $\left\{G_{1}, G_{2}, \ldots, G_{n}\right\}$. O tempo de trabalho $T T_{i}$ de uma gestante $G_{i}$ na maternidade $M_{j}$ é definido neste artigo como sendo a soma do tempo de espera $T E_{i j}$ na fila com o tempo de atendimento $T A_{i j}$. Portanto o objetivo é reduzir o tempo médio de trabalho $T T$ no sistema perinatal. Segue a definição das variáveis de decisão e a formulação do problema:

$$
a_{i j}= \begin{cases}1, & \text { se a gestante } i \text { está admitida na maternidade } j \\ 0, & \text { caso contrário }\end{cases}
$$




$$
\begin{gathered}
b_{i j}= \begin{cases}1, & \text { se a gestante } i \text { está na fila de atendimento da maternidade } j \\
0, & \text { caso contrário }\end{cases} \\
\min (T T)=\min \left(\sum_{j=1}^{m} \sum_{i=1}^{n}\left(T A_{i j} . a_{i j}+T E_{i j} . b_{i j}\right)\right) \quad i=1,2, \ldots, n, \quad j=1,2, \ldots, m(1)
\end{gathered}
$$

O tempo de espera é calculado em função do tempo de atendimento a cada saída de uma gestante da fila, já o tempo de atendimento obedece a uma distribuição exponencial com valores probabilísticos representando o intervalo de 0 a 24 horas. O sistema deve decidir se irá fazer o balanceamento, caso uma gestante não encontre um pronto atendimento ela ficará na fila de espera da maternidade denotado por $G F$. As gestantes atendidas são denotadas por $G A$. À medida que alguma maternidade fica sobrecarregada, o ciclo começa até que todas as gestantes sejam alocadas às maternidades com baixa carga e o sistema esteja equilibrado com base na sua capacidade. Todas as maternidades serão classificadas em ordem crescente e decrescente e agrupadas com base na sua carga. Definimos as maternidades sobrecarregadas $M o$ por $G F>0$ e as maternidades com baixa carga $M u$ por $G F=0$. Essa técnica é descrita pelo Algoritmo 1 .

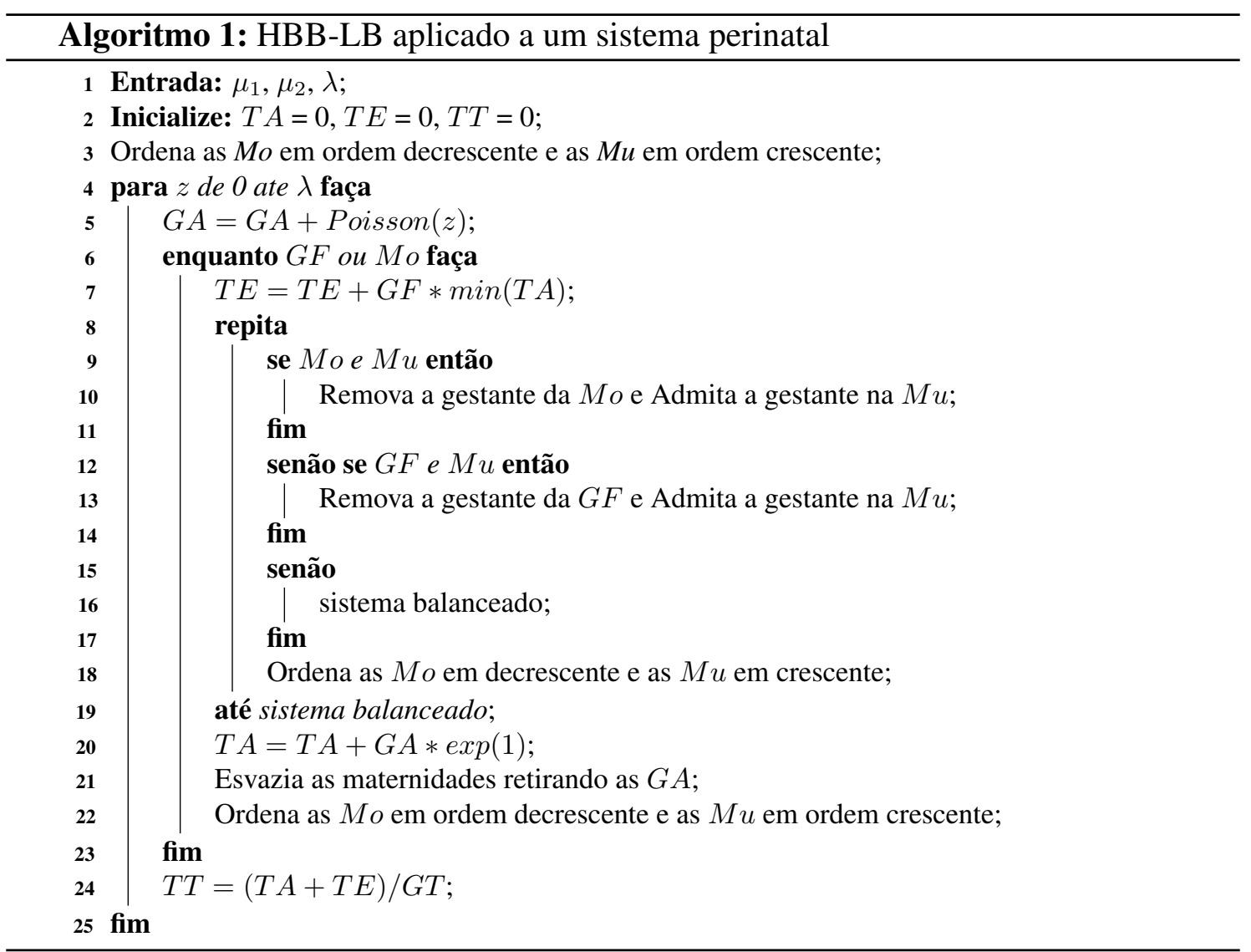




\section{Modelo de Simulação}

A rede perinatal do cenário analisado é apresentada na Figura 2. As chegadas das gestantes as maternidades são modeladas como um processo Poisson com taxas $\lambda_{1} \mathrm{e} \lambda_{2}$. As taxas médias exponenciais de atendimento nas maternidades $M_{1}$ e $M_{2}$ são definidas pelos parâmetros $\mu_{1}$ e $\mu_{2}$, que são determinados em função do número de atendentes e leitos para internação. Na triagem as tomadas de decisões são baseadas na carga das maternidades, os algoritmos de balanceamento de carga foram aplicados com o objetivo de diminuir o tempo médio de trabalho das gestantes que chegam a uma das maternidades.

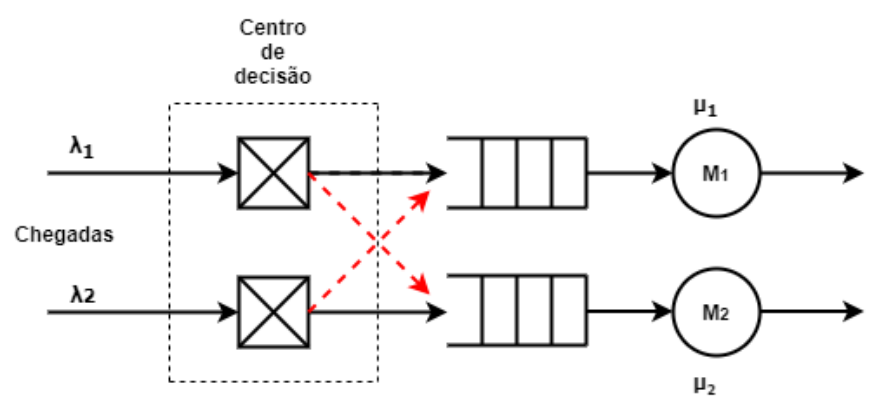

Figura 2. Rede perinatal composta por duas maternidades

Considerando os dados coletados de uma maternidade anônima de Goiânia, pôdese obter a taxa média de chegadas de gestantes por dia baseado no número de atendimentos e procedimentos realizados dentro de certo período. A Tabela 1 apresenta o número de atendimentos realizados na maternidade no período de junho a dezembro de 2018 totalizando um total de 214 dias. As taxas de serviços foram obtidas de acordo com a quantidade de leitos de internação, leitos para partos e leitos de UTI.

Tabela 1. Número médio de procedimentos por dia.

\begin{tabular}{|l|l|l|l|}
\hline Tipo de serviço & Atendimentos & Partos & Entrada de recém-nascidos na UTI + UCIN's \\
\hline Média diária & 31,4 & 2,13 & 3,03 \\
\hline
\end{tabular}

Para a simulação foi considerado que: $\mathrm{M}_{1}$ possui uma quantidade maior de leitos em relação a $\mathbf{M}_{2}$. Portanto, $\mu_{1}>\mu_{2} ; \mathbf{M}_{1}$ possui apenas UTIs neonatais e $\mathbf{M}_{2}$ possui UTIs neonatais e UTIs para mães; Apenas $\mathbf{M}_{2}$ possui Centro Cirúrgico e Ambas possuem Centros de Obstetrícia. Os algoritmos de balanceamento de carga foram aplicados em três etapas: Para decidir a qual maternidade a gestante será encaminhada; Para decidir se a gestante irá pra o CO ou para o CC; Para decidir para qual UTI irão as mães e os recém-nascidos.

\section{Resultados e Discussões}

O algoritmo apresentado neste artigo é comparado com os algoritmos descritos em [Oliveira et al. 2019], utilizando o mesmo cenário, onde os autores analisam diferentes modelos de balanceamento de carga diferenciados por uma estratégia de roteamento com o objetivo de encontrar a melhor política de escalonamento de gestantes.

Em [Oliveira et al. 2019] foram analisados quatro políticas de roteamento: o algoritmo $\mathrm{PR}$, que é um modelo de roteamento não determinístico, e os algoritmos $\mathrm{R}, \mathrm{T}$ e TP 
que são modelos de roteamento determinístico com uma política de roteamento definida por um despachante de tarefas. No algoritmo R, as gestantes são direcionadas para a fila com o menor TT esperado. No algoritmo T busca-se diminuir o tempo de atendimento de todas as gestantes que se encontram no sistema. No algoritmo TP o objetivo é melhorar o desempenho do sistema aumentando a vazão de gestantes nas maternidades.

Inicialmente, foram aplicados os algoritmos de roteamento para as gestantes que chegam ao sistema perinatal, a fim de decidir em qual maternidade, $\mathrm{M}_{1}$ ou $\mathrm{M}_{2}$, elas serão admitidas de acordo com a disponibilidade de recursos. Dado que, $\mathbf{M}_{1}$ possui uma quantidade maior de leito, ficou definido que $\mu_{1}>\mu_{2}$. Uma vez concluída a admissão das gestantes em uma das duas maternidades, utilizamos o algoritmo em cada uma individualmente, de acordo com as gestantes que necessitavam ir para o CC ou para o CO. $\mathrm{M}_{1}$ não possui UTIs para as gestantes, portanto, a taxa de serviços $\mu_{2}$ é relativamente pequena. Caso a gestante necessite de UTI ela deve ser transferida. Já $\mathrm{M}_{2}$ possui UTIs neonatais e UTIs para as mães, portanto, as taxas de serviços $\mu_{1}$ e $\mu_{2}$ estão de acordo com a carga exigida. A Figura 3 apresenta as curvas de TT para admissão ao CC ou CO, os setores mais importantes de uma maternidade, onde ocorrem os partos.

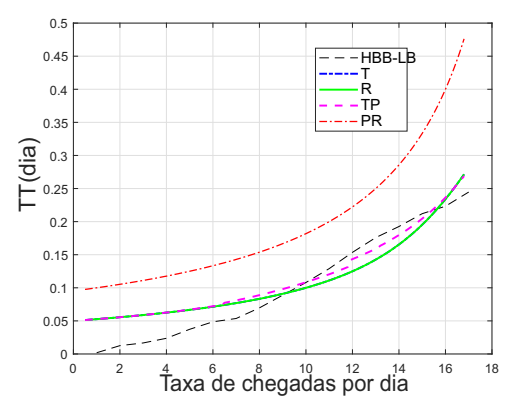

(a) $\mathrm{M}_{1} \operatorname{com} \mu_{1}=20$ e $\mu_{2}=1$

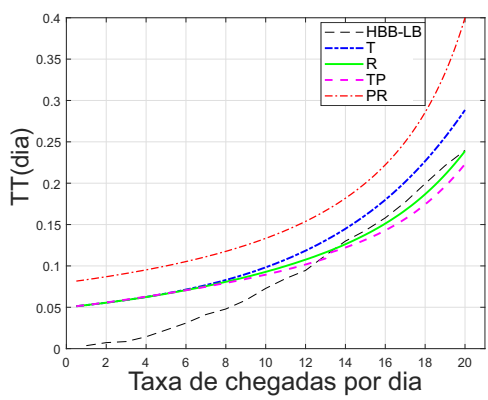

(b) $\mathrm{M}_{2} \operatorname{com} \mu_{1}=20$ e $\mu_{2}=5$

Figura 3. TT para os algoritmos de roteamento HBB-LB, PR, R, T, TP.

O tempo médio de trabalho representa o período médio em dias que as gestantes permanecem internadas na maternidade. No Sistema Único de Saúde (SUS) observou-se, para o período de 2008 a 2012, uma média de permanência para partos normais foi de 2,0 dias, para partos cesáreos 2,6 dias, para partos normais de alto risco 3,2 dias e para partos cesáreos de alto risco 4,2 dias [da Saúde 2012]. O tempo médio de trabalho considerando ambos os partos, em gestações de baixo e alto risco, foi de 2,3 dias nesse período. Os tempos médios de trabalho obtidos com os algoritmos balanceamento são apresentados na Tabela 2.

\section{Tabela 2. Tempo médio de trabalho em dias}

\begin{tabular}{|l|l|l|l|l|l|}
\hline Algoritmo & HBB-LB & PR & R & T & TP \\
\hline Maternidade 1 & 1,38 & 2,53 & 1,53 & 1,64 & 1,42 \\
\hline Maternidade 2 & 1,15 & 2,08 & 1,20 & 1,31 & 1,15 \\
\hline Média do Sistema & 1,26 & 2,30 & 1,36 & 1,47 & 1,28 \\
\hline
\end{tabular}

Embora na situação real se leve em consideração uma quantidade maior de fatores, como o quadro clínico das gestantes, taxas de mortalidade neonatal ou maternal, os resul- 
tados obtidos se mostraram satisfatórios. A média de permanência obtida situou-se entre 1,26 a 2,3 dias na maioria dos algoritmos utilizados, valores melhores que os dados reais apresentados pelo SUS. Conforme esperavamos o algoritmo HBB-LB diminuiu o tempo de permanência das gestantes nas maternidades, além de apresentar melhores resultados em comparação com os dados reais e os outros algoritmos, sendo eficiente à medida que as taxas de chegadas aumentam e quando a quantidade de processamento total é utilizada.

\section{Conclusão}

Neste artigo, foi aplicado um algoritmo de balanceamento de carga para uma rede perinatal com base no comportamento da estratégia de forrageamento das abelhas. Esse algoritmo diminuiu a carga das maternidades sobrecarregadas e reduziu o tempo de espera das gestantes em um sistema perinatal. As gestantes removidas dessas maternidades foram representadas como abelhas, que são as atualizadoras das informações. Comparamos a aplicação do algoritmo baseado na meta-heurística com outras técnicas existentes e os resultados obtidos foram bem próximos, alcançando resultados melhores que os dados reais apresentados pelo SUS. No futuro, planeja-se estender esse tipo de balanceamento considerando as prioridades de atendimento e outros fatores intrínsecos às unidades perinatais.

\section{Agradecimentos}

O presente trabalho foi realizado com apoio da Coordenação de Aperfeiçoamento de Pessoal de Nível Superior-Brasil (CAPES) - Código de Financiamento 001

\section{Referências}

Babu, L. D. D. and Krishna, P. V. (2013). Honey bee behavior inspired load balancing of tasks in cloud computing environments. In Applied Soft Computing Journal, pages 2292-2303. Elsevier.

da Saúde, M. (2012). Ministério da saúde. tabnet win32 3.0: Internações hospitalares do sus - por local de internação - sus. http://tabnet.datasus.gov.br/cgi/ tabcgi.exe?sih/cnv/sxuf. def. Acessado: 14-02-2020.

do Carmo Leal, M., Theme-Filha, M. M., de Moura, E. C., Cecatti, J. G., and Santos, L. M. P. (2015). Atenção ao pré-natal e parto em mulheres usuárias do sistema público de saúde residentes na amazônia legal e no nordeste, Brasil 2010. Revista Brasileira de Saúde Materno Infantil, 15(1):91-104.

Green, L. V. (2005). Capacity planning and management in hospitals. In Operations research and health care, pages 15-41. Springer.

Karaboga, D. (2005). An idea based on honey bee swarm for numerical optimization. In Tech. Report TRO6. Erciyes University, Engineering Faculty, Computer Engineering Department.

Oliveira, R. B. O. d., Rocha, F. G. C., and Silva, M. R. P. (2019). Avaliação de modelos de balanceamento de carga aplicados a um sistema perinatal. LI Simpósio Brasileiro de Pesquisa Operacional.

Pehlivan, C. (2014). Design and flow control of stochastic health care networks without waiting rooms: A perinatal application. $\mathrm{PhD}$ thesis, Ecole Nationale Supérieure des Mines de Saint-Etienne. 